\title{
Once-Weekly Teriparatide Treatment Prevents Microdamage Accumulation in the Lumbar Vertebral Trabecular Bone of Ovariectomized Cynomolgus Monkeys
}

\author{
Shingo Yoshitake ${ }^{1} \cdot$ Tasuku Mashiba $^{1} \cdot$ Mitsuru Saito $^{2} \cdot$ Ryuji Fujihara $^{1} \cdot$ Ken Iwata $^{1} \cdot$ Ryoko Takao-Kawabata $^{3}$. \\ Tetsuji Yamamoto ${ }^{1}$
}

Received: 25 October 2018 / Accepted: 26 November 2018 / Published online: 18 December 2018

(c) The Author(s) 2018

\begin{abstract}
The effect of teriparatide treatment on microdamage accumulation has yet to be examined in animal studies. The purpose of this study was to investigate the effect of once-weekly teriparatide treatment on bone microdamage accumulation and the relationship between microdamage parameters and bone mass, architecture, turnover, and collagen cross-linking in the lumbar vertebral trabecular bone of ovariectomized (OVX) cynomolgus monkeys. Female monkeys were divided into four groups ( $n=18-20$ per group): (1) SHAM group, (2) OVX group, (3) OVX with $1.2 \mu \mathrm{g} / \mathrm{kg}$ once-weekly teriparatide group (LOW group), (4) OVX with $6.0 \mu \mathrm{g} / \mathrm{kg}$ once-weekly teriparatide group (HIGH group). After 18 months, all animals were double-labeled with calcein for histomorphometry. L3 and L7 lumbar vertebrae were harvested and analyzed for differences in histomorphometry, microdamage, and collagen cross-linking. The iliac crest was also analyzed for differences in bone turnover. In the OVX group, cancellous bone mass was reduced and microdamage accumulation was increased as compared with the SHAM control. Once-weekly teriparatide at both doses prevented the decrease in bone mass and increase in microdamage accumulation, and improved the distribution of collagen cross-linkage types. Regression analyses indicated that decreased microdamage accumulation was associated with reduced non-enzymatic cross-link pentosidine rather than increased cancellous bone mass or enzymatic cross-links. These findings suggest that once-weekly teriparatide treatment decreases microdamage accumulation by recovering the balance in collagen cross-links.
\end{abstract}

Keywords Teriparatide $\cdot$ Monkey $\cdot$ Bone histomorphometry $\cdot$ Bone microdamage $\cdot$ Collagen cross-links

\section{Introduction}

Teriparatide is an anabolic agent used to treat patients with osteoporosis who have an especially high risk of fracture $[1,2]$. The anabolic effect of teriparatide on cancellous bone has been demonstrated using iliac crest biopsies from

Tasuku Mashiba

task@med.kagawa-u.ac.jp

1 Department of Orthopedic Surgery, Faculty of Medicine, Kagawa University, 1750-1 Ikenobe, Miki-cho, Kita-gun, Kagawa 761-0793, Japan

2 Department of Orthopaedic Surgery, Jikei University School of Medicine, 3-25-8, Nishi-Shinbashi, Minato-ku, Tokyo 105-8461, Japan

3 Pharmaceuticals Research Center, Asahi Kasei Pharma Corporation, 632-1 Mifuku, Izunokuni, Shizuoka 410-2321, Japan postmenopausal women [3-5] and in animal experiments following ovariectomy [6-9]. Teriparatide accelerates not only bone formation but also bone resorption, leading to increased bone remodeling.

Although patients are routinely treated with daily teriparatide injections, a once-weekly treatment regime for teriparatide is already used in Japan [10] and South Korea [11] to treat osteoporotic patients with high fracture risk. Weekly teriparatide treatment can reduce the incidence of osteoporotic vertebral fractures by $78 \%$ in postmenopausal women with prevalent vertebral fractures [10]. In ovariectomized cynomolgus monkeys, we have recently reported that onceweekly teriparatide injections for 18 months can increase the strength of the lumbar vertebrae through a gain in cancellous bone mass and a recovery in the balance of collagen cross-links, with a higher content of enzymatic immature and mature cross-links and lower content of non-enzymatic advanced glycation end product cross-links [6]. 
Although collagen cross-linking is important for the material properties of bone [6,12-15], several other factors also contribute to its material properties [4, 7-9, 16-19]. For instance, physiological loading can cause an accumulation of bone microdamage through bone fatigue [19-21], which in turn can lead to increased bone fragility $[5,14,16,18$, 19]. The overall microdamage burden is a balance between the amount of damage that is produced and the amount that is repaired $[18,19,22,23]$ through bone remodeling [19, 22]. As such, increased bone remodeling by teriparatide treatment would be expected to accelerate microdamage repair, and thereby decrease microdamage accumulation. However, to our knowledge, few studies have investigated the effect of teriparatide on bone microdamage accumulation [5]. Furthermore, there are no animal or clinical data on the effects of once-weekly teriparatide treatment on microdamage accumulation.

The primary purpose of the present study was to investigate the effect of once-weekly teriparatide treatment on bone microdamage accumulation in the lumbar vertebral trabecular bone of ovariectomized (OVX) cynomolgus monkeys. The secondary purpose was to examine the relationship between microdamage parameters and bone mass, architecture, turnover, and collagen cross-linking. In this study, histomorphometry was used to monitor changes in trabecular bone mass, architecture, turnover, and microdamage. Although the results of collagen cross-links have already been published previously [6], the association of cross-links with microdamage parameters was additionally examined.

\section{Materials and Methods}

\section{Animals and Experimental Design}

The animals and experimental design of this study have been previously described [6]. Skeletally mature 80 female cynomolgus monkeys (mean age $\pm \mathrm{SD}, 12.0 \pm 1.5$ years) were obtained from C.V. Universal Fauna (Jakarta Timur, Indonesia). Monkeys were housed under controlled conditions at $26{ }^{\circ} \mathrm{C} \pm 2{ }^{\circ} \mathrm{C}$ with a 12 -h light-dark cycle, and were given free access to water and approximately $108 \mathrm{~g}$ of commercial food containing $1.4 \%$ calcium and $0.6 \%$ phosphorus (Harlan Sprague Dawley Inc., Indianapolis, Indiana, U.S.) once daily. Interventions were commenced after monkeys had acclimated for 6 weeks.

Seventy-seven monkeys (body weight at grouping, 2.06-3.48 kg) exhibiting normal behavior during the acclimation period were selected and divided into four groups ( $n=18-20)$ based on body weight, spine bone mineral density (BMD), and age, as follows: (1) SHAM group, (2) OVX group, (3) OVX with low-dose teriparatide treatment group (LOW group), and (4) OVX with high-dose teriparatide treatment group (HIGH group). Ovariectomy was performed 1 week before additional treatment. SHAM and OVX groups were subcutaneously administered onceweekly with $0.1 \%$ saline containing bovine serum albumin as a vehicle, whereas the LOW and HIGH groups received once-weekly teriparatide acetate (Asahi Kasei Pharma Corp., Tokyo, Japan) at doses of $1.2 \mu \mathrm{g} / \mathrm{kg}$ and $6.0 \mu \mathrm{g} / \mathrm{kg}$ body weight, respectively. Experiments were performed for 18 months. Teriparatide dosages were chosen based on previous reports [24]. For bone histomorphometry, all animals were double-labeled with calcein $(4 \mathrm{mg} / \mathrm{kg}$; Dojindo Laboratories, Kumamoto, Japan) through intravenous injections on days 21 and 7 before sacrifice. Monkeys were sacrificed by an overdose of pentobarbital sodium (Tokyo Chemical Industry Co., Ltd., Tokyo, Japan).

\section{Bone Preparation}

After sacrifice at 18 months, the left iliac crest, and the third and seventh lumbar vertebrae (L3, L7) were harvested and freed from adherent soft tissues. We excluded 25 specimens of the iliac crest because of inadequate tissue quantity. Iliac crest samples were fixed with $70 \%$ ethanol and embedded in methyl methacrylate-based plastic. Cancellous bone fractions of the L3 vertebral bodies were cleaned of bone marrow, frozen in liquid nitrogen, and pulverized with liquid nitrogen, as previously reported [12, 25]. L7 vertebrae were fixed with $70 \%$ ethanol, stained with $1 \%$ basic fuchsin for 3 days, and then embedded in methyl methacrylate-based plastic.

\section{Bone Histomorphometry}

Samples of the iliac crest and L7 vertebrae were used for dynamic and static histomorphometric analyses, respectively. Embedded iliac crest samples were cut to 5- $\mu \mathrm{m}$-thick unstained coronal sections using a microtome. L7 vertebrae were sectioned around the mid-sagittal portion with a diamond saw and ground to $100 \mu \mathrm{m}$ thickness for structural evaluation and microdamage analysis of cancellous bone.

Histomorphometric measurements were performed using a semi-automated digitizing image analyzer, consisting of a light or epifluorescence microscope at $\times 100$ magnification and a digitizing pad connected to a computer with histomorphometric software (System Supply Co., Nagano, Japan). Polarized light was applied to identify the lamellar structure and trabecular packets. All measurements were carried out blindly by one histomorphometrist. A square $(5 \times 5 \mathrm{~mm})$ area at the center of the cancellous bone in the L7 vertebra was measured for trabecular bone structure and microdamage analyses.

Microdamage in bone was defined as linear shapes of basic fuchsin stain located in the bone matrix and that 
could be obviously discriminated from canaliculi or vascular canals. Classically, microscopic cracks are defined as having sharp edges; however, we included lamellar debonding cracks without sharp edges to obtain a sufficient number of cracks for analysis, as based on a previous report in this field [18] (Fig. 1). All microdamage has some depth of field and permeation of the stain into the crack walls $[18,23]$. We measured the mean crack length $(\mathrm{Cr} . \mathrm{Le}, \mu \mathrm{m})$, crack density $(\mathrm{Cr} . \mathrm{Dn}=\mathrm{Cr} . \mathrm{N} / \mathrm{B} . \mathrm{Ar}$, $\# / \mathrm{mm}^{2}$ ), and crack surface density (Cr.S.Dn $=\mathrm{Cr} . \mathrm{N} \times \mathrm{Cr}$. Le/B.Ar $\mu \mathrm{m} / \mathrm{mm}^{2}$ ). For each animal, two sections of the L7 vertebra were measured.

For trabecular structural analyses, trabecular bone volume (BV/TV, \%), trabecular thickness (Tb.Th, $\mu \mathrm{m})$, tra-

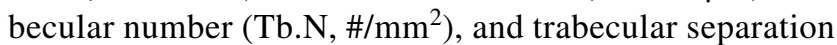
(Tb.Sp, $\mu \mathrm{m}$ ) were measured.

The following parameters of the iliac crest were measured as trabecular bone-labeled parameters: single-labeled surface (sLS/BS, \%), double-labeled surface (dLS/BS, \%), mineralized surface (MS/BS, \%), mineral apposition rate (MAR, $\mu \mathrm{m} /$ day), and bone formation rate (BFR/BS, $\mu \mathrm{m}^{3} /$ $\mu \mathrm{m}^{2} /$ year and BFR/BV, \%). For these measures of conventional histomorphometry, one section of the L7 vertebra was measured for each animal.

The nomenclature and symbols used are in accordance with the report of the Histomorphometry Nomenclature Committee of the American Society for Bone and Mineral Research [26].

\section{Measurement of Collagen Cross-Links}

The measurement of collagen cross-links was carried out as previously described [25]. Briefly, bone powder was demineralized with $0.5 \mathrm{M}$ EDTA in $50 \mathrm{mM}$ Tris buffer (pH 7.4)

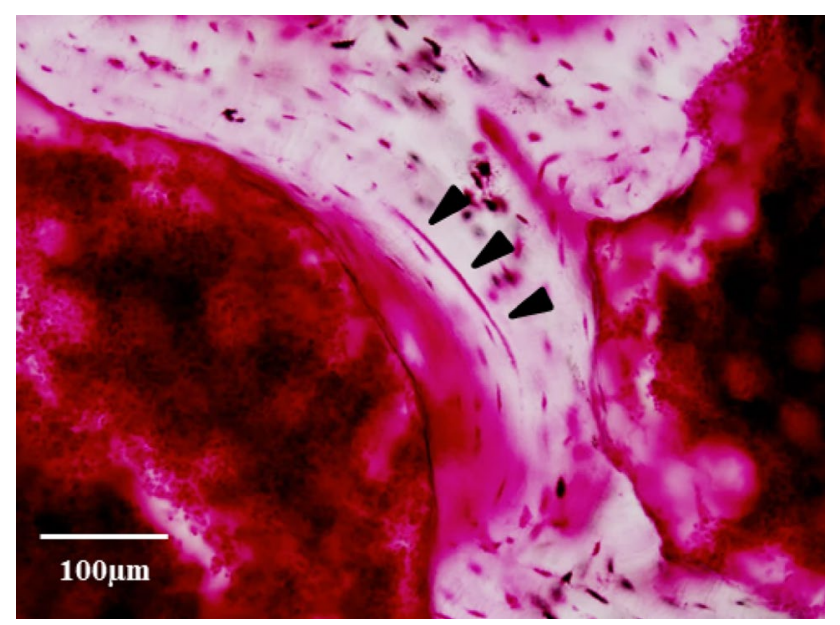

Fig. 1 Photograph of a lamellar debonding microcrack in the trabecular bone of an L7 vertebra (arrow). Stained en bloc with basic fuchsin. Original magnification, $\times 100$ for $96 \mathrm{~h}$ at $4{ }^{\circ} \mathrm{C}$. Demineralized bone residue was suspended in potassium phosphate buffer ( $\mathrm{pH}$ 7.6) and reduced at $37{ }^{\circ} \mathrm{C}$ with sodium borohydride (Sigma-Aldrich, St Louis, MO, USA). The specimens were hydrolyzed in $6 \mathrm{~N} \mathrm{HCl}$ at $110{ }^{\circ} \mathrm{C}$ for $24 \mathrm{~h}$. Hydrolysates were then analyzed for the content of cross-links and hydroxyproline on a Shimadzu LC9 highperformance liquid chromatography (HPLC) system fitted with a cation exchange column $(0.9 \times 10 \mathrm{~cm}$, Aa pack-Na; Jasco, Tokyo, Japan) and linked to an online fluorescence flow monitor (RF10AXL; Shimadzu, Kyoto, Japan). It was assumed that collagen weighed 7.5 times the measured hydroxyproline weight, based on a molecular weight of $300,000 \mathrm{Da}$ [25]. The resulting data were used to calculate cross-link values as $\mathrm{mol} / \mathrm{mol}$ of collagen. Reducible immature cross-links (deH-dihydroxylysinonorleucine, deHhydroxylysinonorleucine, and deH-lysinonorleucine) were identified and quantified as their reduced forms (DHLNL, HLNL, LNL, respectively). Our established HPLC system allows us to determine enzymatic and non-enzymatic crosslink content within a linear range of $0.2-600 \mathrm{pmol}$ in bone specimens. Reducible cross-links and common amino acids, such as hydroxyproline, are detected with post-column derivatization using o-phthalaldehyde, whereas non-reducible cross-links (Pyr: pyridinoline; Dpyr: deoxypyridinoline) and pentosidine are detected by natural fluorescence. The units for enzymatic and non-enzymatic cross-links are mol/ $\mathrm{mol}$ and $\mathrm{mmol} / \mathrm{mol}$ of collagen, respectively. The content of pentosidine was determined as per the method of Tang et al. [14].

The following parameters for L3 were measured as collagen parameters: immature + mature pyridinium cross-links (DHNL + HLNL + LNL + Pyr + Dpyr, mol/ mol of collagen), mature cross-links rates ([Pyr $+\mathrm{Dpyr}] /$ $[\mathrm{DHNL}+\mathrm{HLNL}+\mathrm{LNL}])$, and pentosidine $(\mathrm{mmol} / \mathrm{mol}$ of collagen).

\section{Statistical analysis}

All values are expressed as the mean \pm standard deviation (SD). Differences among the four groups were tested by oneway analysis of variance (ANOVA). If a significant difference was found, Tukey test was used for multiple comparisons to clarify differences among two groups. Spearman's rank correlation coefficient was used to select a parameter to explain microcrack accumulation from trabecular bone structural parameters, trabecular bone-labeled parameters, and collagen cross-link parameters in the three ovariectomized groups (OVX $+\mathrm{LOW}+\mathrm{HIGH})$. All $p$ values $<0.05$ were considered significant.

All statistical analyses were performed with EZR (Saitama Medical Center, Jichi Medical University, Saitama, 
Japan), which is a graphical user interface for $\mathrm{R}$ (The $\mathrm{R}$ Foundation for Statistical Computing, Vienna Austria).

\section{Results}

\section{Trabecular Bone Structural Parameters}

$\mathrm{BV} / \mathrm{TV}$ and Tb.Th were significantly lower in the OVX group than in the SHAM group $(p<0.05)$. Among the OVX treatment groups, BV/TV and Tb.Th were significantly higher in the LOW group than in the OVX group $(p<0.05)$, and Tb.Th in the HIGH group was significantly higher than that in the OVX group $(p<0.05)$ (Fig. 2a). No significant differences in Tb.N or Tb.Sp were found among the groups. There were no significant changes between the LOW and HIGH groups or between the SHAM group and either of teriparatide treatment groups (Table 1).

\section{Microdamage Parameters}

Cr.Le was not significantly different among the four groups. Cr.Dn and Cr.S.Dn were significantly higher in the OVX group than in the other groups $(p<0.05)$, with no significant differences among the SHAM, LOW, and HIGH groups (Fig. 2b). There were no significant differences between the LOW and HIGH groups (Table 2).

\section{Dynamic Parameters}

There were no significant differences for any of the dynamic histomorphometric parameters obtained from the iliac crest based on calcein labels among the four groups (Table 3).
Fig. 2 Effect of once-weekly teriparatide treatment on trabecular bone mass, microcrack density, and collagen cross-links in ovariectomized cynomolgus monkeys. a Trabecular bone volume. b Microcrack density. c Total amount of enzymatic mature and immature collagen cross-links. d Pentosidine content. $* p<0.05$ (a) BV/TV

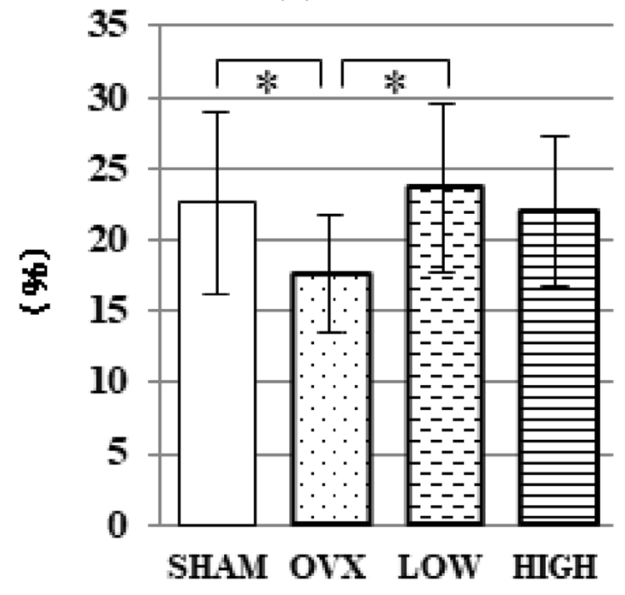

(c) $\mathbf{I M}+\mathbf{M}$

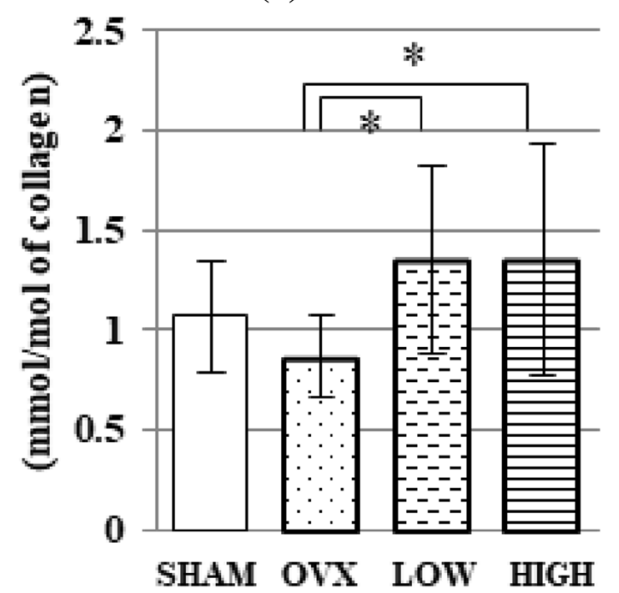

(b) Cr.Dn

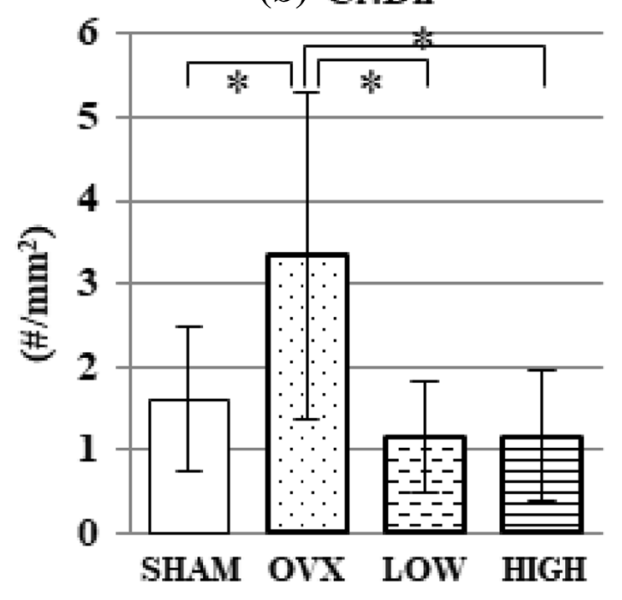

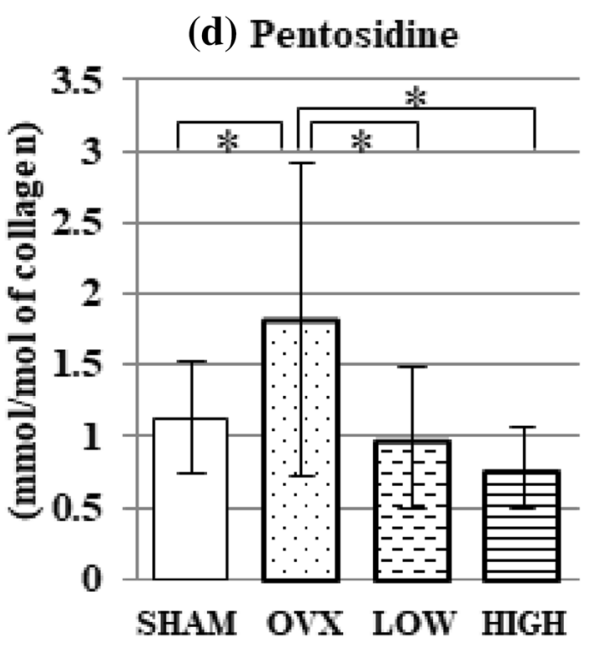


Table 1 Structural parameters of L7 trabecular bone

\begin{tabular}{lcccc}
\hline & SHAM $(n=19)$ & OVX $(n=18)$ & LOW $(n=20)$ & HIGH $(n=20)$ \\
\hline BV/TV $(\%)$ & $22.6 \pm 6.4$ & $17.6 \pm 4.1^{*}$ & $23.7 \pm 5.9^{\#}$ & $22.0 \pm 5.2$ \\
Tb.Th $(\mu \mathrm{m})$ & $139.9 \pm 32.9$ & $113.0 \pm 17.7^{*}$ & $144.9 \pm 19.7^{\#}$ & $137.5 \pm 25.9^{\#}$ \\
Tb.N $(/ \mathrm{mm})$ & $1.62 \pm 0.27$ & $1.55 \pm 0.25$ & $1.64 \pm 0.31$ & $1.60 \pm 0.26$ \\
Tb.Sp $(\mu \mathrm{m})$ & $496.7 \pm 120.4$ & $553.3 \pm 146.9$ & $489.8 \pm 137.6$ & $503.1 \pm 101.4$ \\
\hline
\end{tabular}

Data are expressed as mean \pm SD

${ }^{*} p<0.05$ versus SHAM; ${ }^{*} p<0.05$ versus OVX

\begin{tabular}{lcccc}
\hline & SHAM $(n=19)$ & OVX $(n=18)$ & LOW $(n=20)$ & HIGH $(n=20)$ \\
\hline Cr.Le $(\mu \mathrm{m})$ & $63.4 \pm 10.5$ & $57.2 \pm 7.9$ & $65.2 \pm 12.5$ & $61.8 \pm 19.3$ \\
Cr.Dn $\left(\# / \mathrm{mm}^{2}\right)$ & $1.61 \pm 0.86$ & $3.33 \pm 1.97^{*}$ & $1.16 \pm 0.67^{\#}$ & $1.17 \pm 0.78^{\#}$ \\
Cr.S.Dn $\left(\mu \mathrm{m} / \mathrm{mm}^{2}\right)$ & $102.7 \pm 61.6$ & $185.0 \pm 101.8^{*}$ & $72.5 \pm 40.2^{\#}$ & $77.4 \pm 52.6^{\#}$ \\
\hline
\end{tabular}

Data are expressed as mean $\pm \mathrm{SD}$

${ }^{*} p<0.05$ versus SHAM; ${ }^{\#} p<0.05$ versus OVX

\begin{tabular}{lcccc}
\hline & SHAM $(n=10)$ & OVX $(n=14)$ & LOW $(n=17)$ & HIGH $(n=12)$ \\
\hline sLS/BS $(\%)$ & $12.48 \pm 7.10$ & $10.35 \pm 6.56$ & $10.17 \pm 5.64$ & $10.30 \pm 5.90$ \\
dLS/BS $(\%)$ & $6.92 \pm 6.47$ & $6.51 \pm 5.68$ & $9.00 \pm 8.21$ & $9.64 \pm 8.39$ \\
MS/BS $(\%)$ & $13.16 \pm 8.86$ & $11.69 \pm 7.41$ & $14.08 \pm 10.26$ & $14.79 \pm 10.58$ \\
MAR $(\mu \mathrm{m} /$ day $)$ & $0.607 \pm 0.223$ & $0.601 \pm 0.132$ & $0.646 \pm 0.172$ & $0.689 \pm 0.242$ \\
BFR/BS $\left(\mu \mathrm{m}^{3} / \mu^{2} /\right.$ year $)$ & $31.5 \pm 22.7$ & $26.8 \pm 16.7$ & $36.7 \pm 31.0$ & $40.9 \pm 33.9$ \\
BFR/BV $(\%)$ & $59.6 \pm 46.4$ & $57.0 \pm 35.5$ & $66.3 \pm 62.2$ & $64.6 \pm 48.1$ \\
\hline
\end{tabular}

Data are expressed as mean $\pm \mathrm{SD}$

\begin{tabular}{lcccc}
\hline & SHAM $(n=19)$ & OVX $(n=18)$ & LOW $(n=19)$ & HIGH $(n=20)$ \\
\hline $\begin{array}{l}\text { IM }+\mathrm{M} \\
\text { (mol/mol of collagen) }\end{array}$ & $1.069 \pm 0.283$ & $0.867 \pm 0.206$ & $1.354 \pm 0.474^{\#}$ & $1.353 \pm 0.582^{\#}$ \\
Mature cross-link ratio & $0.207 \pm 0.073$ & $0.233 \pm 0.103$ & $0.183 \pm 0.096$ & $0.175 \pm 0.057$ \\
$\begin{array}{l}\text { Pentosidine } \\
\text { (mmol/mol of collagen) }\end{array}$ & $1.128 \pm 0.387$ & $1.816 \pm 1.094^{*}$ & $0.987 \pm 0.495^{\#}$ & $0.780 \pm 0.293^{\#}$ \\
\hline
\end{tabular}

Data are expressed as mean $\pm \mathrm{SD}$

$I M$ immature cross-links, the sum of DHLNL, HLNL, and LNL, $M$ mature pyridinium cross-links, the sum of Pyr and Dpyr

${ }^{*} p<0.05$ versus SHAM; ${ }^{*} p<0.05$ versus OVX

\section{Collagen Parameters}

The OVX group showed a non-significant decrease in the content of enzymatic immature + mature pyridinium crosslinks and a significant increase in the content of pentosidine as compared with the SHAM group. The content of enzymatic immature + mature cross-links was significantly higher in both the LOW and HIGH groups as compared with the OVX group $(p<0.05)$ (Fig. 2c). Pentosidine content was significantly lower in the LOW and HIGH groups as compared with the OVX group $(p<0.05)$ (Fig. $2 d)$. The mature cross-link ratio was not significantly different among the four groups. There were no significant differences between the SHAM group and the teriparatide treatment groups, or between the LOW and HIGH groups (Table 4).

\section{Correlation Between Microdamage Parameters and Other Parameters}

$\mathrm{BV} / \mathrm{TV}, \mathrm{Tb}$. Th, and immature + mature pyridinium crosslinks were significantly and slightly negatively correlated with Cr.Dn and Cr.S.Dn $(p<0.05)$ (Fig. 3a, b). In contrast, 


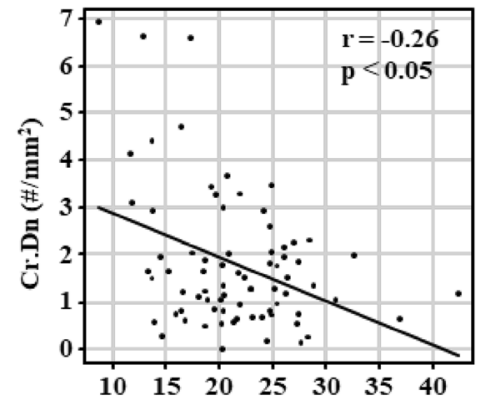

(a) BV/TV (\%)

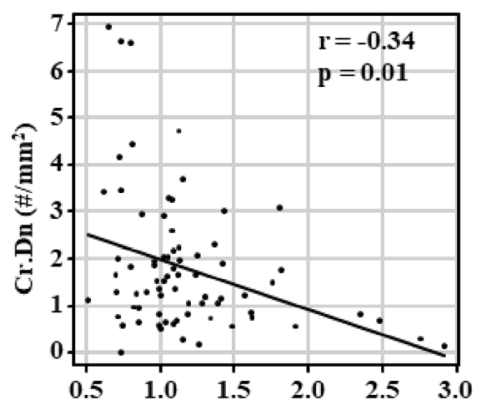

(b) Immature+mature pyridinium cross-links ( $\mathrm{mmol} / \mathrm{mol}$ of collagen)

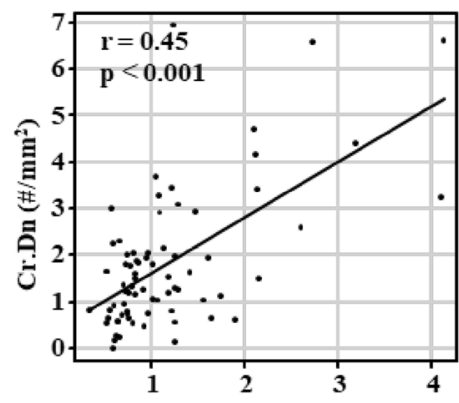

(c) Pentosidine ( $\mathrm{mmol} / \mathrm{mol}$ of collagen)
Fig. 3 Regression analysis of microcrack density (Cr.Dn) with trabecular bone mass (BV/TV) and collagen cross-links. Increased Cr.Dn was significantly associated with decreased BV/TV (a), a decreased

immature + mature pyridinium cross-links (Cr.Le) were significantly and slightly positively correlated with microdamage parameters $(p<0.05)$. Among all the parameters, pentosidine was most strongly correlated with increased Cr.Dn and Cr.S.Dn $(p<0.001)$ (Table 5; Fig. 3c).

\section{Discussion}

In this study, we investigated the effects of once-weekly teriparatide treatment on trabecular structure, bone turnover, microdamage accumulation, and collagen cross-links in the cancellous bone of ovariectomized cynomolgus monkeys. Our results demonstrate that teriparatide increases trabecular bone by thickening the trabeculae and decreasing microdamage accumulation, probably due to a favorable shift in the balance of collagen cross-links (increased enzymatic amount of enzymatic immature and mature collagen cross-links $(\mathrm{IM}+\mathrm{M}, \mathbf{b})$ and an increased amount of non-enzymatic cross-link pentosidine (c)

immature + mature collagen cross-links and decreased nonenzymatic collagen cross-links, pentosidine) in the vertebral cancellous bone. Although several animal studies have shown that teriparatide treatment can increase bone mass and improve trabecular architecture by accelerated bone formation [6-9, 27], few studies have evaluated the effects of teriparatide treatment on microdamage accumulation [5]. To the best of our knowledge, this is the first animal study to investigate the effect of teriparatide treatment on microdamage accumulation in vertebral trabecular bone.

In this study, the OVX group had significantly higher microdamage accumulation than the SHAM group. This was unexpected, because microdamage is thought to be lowered by damage repair strategies that are elevated in response to bone remodeling following ovariectomy [18, 19, 23]. There are several possible explanations for this phenomenon. First, as shown in our results, the increase in bone
Table 5 Correlation of microdamage parameters with the trabecular structural, dynamic, and collagen crosslink parameters

\begin{tabular}{|c|c|c|c|c|c|c|}
\hline & \multicolumn{2}{|l|}{ Cr.Le } & \multicolumn{2}{|l|}{ Cr.Dn } & \multicolumn{2}{|c|}{ Cr.S.Dn } \\
\hline & $r$ & $p$ & $r$ & $p$ & $r$ & $p$ \\
\hline $\mathrm{BV} / \mathrm{TV}$ & -0.03 & 0.85 & -0.26 & $<0.05$ & -0.29 & 0.03 \\
\hline Tb.Th & 0.16 & 0.23 & -0.38 & 0.003 & -0.38 & 0.003 \\
\hline Tb.N & 0.14 & 0.28 & 0.11 & 0.42 & 0.07 & 0.61 \\
\hline $\mathrm{Tb} . \mathrm{Sp}$ & 0.09 & 0.5 & 0.3 & 0.83 & 0.06 & 0.64 \\
\hline sLS & -0.05 & 0.77 & 0.09 & 0.58 & 0.09 & 0.58 \\
\hline dLS & 0.15 & 0.36 & 0.10 & 0.54 & 0.16 & 0.31 \\
\hline MS/BS & 0.12 & 0.45 & 0.14 & 0.39 & 0.20 & 0.22 \\
\hline MAR & -0.12 & 0.45 & 0.12 & 0.45 & 0.13 & 0.42 \\
\hline $\mathrm{BFR} / \mathrm{BS}$ & 0.13 & 0.43 & 0.14 & 0.38 & 0.19 & 0.22 \\
\hline BFR/BV & 0.08 & 0.62 & 0.24 & 0.13 & 0.29 & 0.06 \\
\hline $\mathrm{IM}+\mathrm{M}$ & 0.33 & 0.01 & -0.34 & 0.01 & -0.27 & 0.04 \\
\hline M/IM & -0.28 & 0.04 & 0.14 & 0.31 & 0.08 & 0.58 \\
\hline Pentosidine & -0.24 & 0.08 & 0.45 & $<0.001$ & 0.42 & $<0.001$ \\
\hline
\end{tabular}

IM Immature cross-links, the sum of DHLNL, HLNL, and LNL, $M$ Mature pyridinium cross-links, the sum of Pyr and Dpyr 
turnover by ovariectomy caused a reduction in trabecular bone volume, which in turn could lead to stress concentration on the trabeculae. Therefore, the production of microdamage might exceed the rate of damage repair. Second, the increase in turnover by ovariectomy may not be long-lasting in cynomolgus monkeys $[9,28]$. The present study found no significant difference in any of the dynamic parameters between the OVX and SHAM groups, suggesting that the increase in bone turnover by ovariectomy returns to normal levels within 18 months after surgery. This time-dependent reduction in bone turnover may accelerate microdamage accumulation by lower rates of damage repair. Finally, the elevated levels of pentosidine, which is representative of the production of advanced glycation end products (AGEs), in the OVX group might increase bone fragility [6] and rates of microdamage [14]. As reported previously, the amount of pentosidine or AGEs increases after OVX due to oxidative stress induced by estrogen deficiency, even in situations of high bone turnover [29].

The most striking finding of this study was that onceweekly teriparatide treatment decreased microdamage accumulation in the vertebral cancellous bone, and that this reduction was associated with a favorable shift in the balance of collagen cross-links. As previously reported [6], once-weekly teriparatide treatment increased the total content of enzymatic immature and mature cross-links, which contributed to increased vertebral bone strength, and decreased AGE cross-linking pentosidine, which is reported to accumulate with tissue aging and time-dependent oxidation and glycation, leading to microdamage and bone fragility. In the current study, both increased immature + mature pyridinium cross-links and decreased pentosidine were significantly associated with decreased Cr.Dn or Cr.S.Dn. The increase in immature + mature pyridinium cross-links means an increase in the enzymatic cross-links of collagen, and this can have a positive effect on the mechanical properties of bone within a beneficial range, without brittleness [13, 15]. Indeed, in our previous study [6], the amount of immature and mature collagen cross-links was significantly and positively correlated with ultimate load, stiffness, and breaking energy of the L3 vertebra. In that study as well, pentosidine content was significantly negatively correlated with lumbar vertebral stiffness. In the current study, which examines the relationship between microdamage accumulation and trabecular bone mass, architecture, bone turnover, and collagen cross-links, pentosidine content was most strongly associated with an increased accumulation of microdamage. This may imply that increased pentosidine content is a key factor contributing to the bone brittleness or fragility that causes microdamage in bone.

Theoretically, increased bone turnover can explain how teriparatide reduces the accumulation of microdamage, as it enhances damage repair through targeted bone remodeling
$[18,19,22,23]$. In the current study, however, there were no relationships between microdamage accumulation and any of the bone turnover parameters. This may be because of the 18-month duration of teriparatide treatment. Although increased bone turnover caused by once-daily teriparatide treatment was most evident in the early phase of treatment, its effect becomes weaker with time and finally reaches a plateau over a longer treatment period [30-32]. Our results suggest that 18 months of once-weekly teriparatide treatment is too long to detect improvements in bone turnover based on histomorphometric dynamic parameters. An evaluation of bone dynamic parameters and microdamage at an earlier phase of treatment might provide more important information regarding the relationship between bone turnover and microdamage accumulation.

In this study, no significant differences were found for any of the parameters between the LOW and HIGH groups. The dosage of teriparatide in the LOW group is the clinical dosage of once-weekly teriparatide treatment for patients with osteoporosis in Japan. The dosage in the HIGH group was five times the clinical dosage, and this was decided according to the FDA guideline issued in 1994. Our findings suggest that the clinical dosage of teriparatide is effective and appropriate for a once-weekly dosing regimen. Several animal studies have demonstrated the dose-dependent anabolic effects of daily teriparatide treatment on bone $[8,9$, 33]. The study reported by Jerome et al. [9], in which ovariectomized cynomolgus monkeys were treated with daily teriparatide injections at 1 or $5 \mu \mathrm{g} / \mathrm{kg}$ for 18 months, found dose-dependent positive effects of teriparatide on cancellous bone mass and architecture in both the axial and appendicular skeleton. This is in contrast with the findings of our previous [6] and current studies, in which the same animals were treated with once-weekly teriparatide injection at 1.2 or $6 \mu \mathrm{g} / \mathrm{kg}$ for 18 months. A dose-dependent effect of onceweekly teriparatide treatment may be less evident even in monkeys treated with a lower dose of teriparatide as compared with daily treatment. Future studies should examine the relationship between the anabolic effect on bone and the treatment regimen of teriparatide.

One clinical study exists regarding the effects of teriparatide treatment on microdamage accumulation using iliac crest biopsy [5]. Dobnig et al. [5] showed that daily administration of teriparatide for 2 years reduced microdamage accumulation in postmenopausal osteoporotic patients previously treated with alendronate. In their study, however, teriparatide did not reduce microdamage in bisphosphonatenative patients. There were two possible reasons for the difference between the study by Dobnig and colleagues and our study. One is the differences in the evaluated specimens: The lumbar vertebrae used in our study may experience a higher microdamage burden during weight bearing or daily activities than the iliac crest. Furthermore, the response to 
anti-osteoporotic treatments may be greater in the lumbar vertebra than in the ilium because the lumbar vertebrae are usually more sensitive to changes in BMD than other skeletal structures in clinical situations [2, 10]. Another reason is the difference in the histological definition of microcracks: we expanded our definition of microcracks according to a previous report [18] to include microcracks without sharp crack edges. This presumably led to the identification of a higher number of cracks and, by extension, increased the sensitivity of the assay within a limited measurable area on histological sections. Indeed, it would be difficult to detect significant changes in microdamage in response to treatment when there are few measurable cracks before treatment. Therefore, it is possible that more microcracks overall were detected in the current study.

Our study has several limitations. First, we were not able to detect significant correlations between microdamage and mechanical properties (data not shown). This contrasted with a previous study that found significant correlations between collagen cross-links and mechanical strength with once-weekly teriparatide treatment [6]. Microdamage may not be as strongly associated with the mechanical properties of bone as collagen cross-links. Second, histomorphometric dynamic parameters regarding bone turnover were not measured in the vertebrae but in the iliac crest. This is because this experiment was performed several years ago. We assessed dynamic parameters soon after sacrifice to avoid loss of the label over a long-term fixation. Lumbar vertebral dynamic parameters obtained soon after sacrifice may provide better information about the relationship between bone turnover and microdamage accumulation. Third, specimen preparation for microdamage analysis was performed on specimens stored for several years in $70 \%$ ethanol. Longterm fixation might affect the material properties of bone and increase the production of microdamage through sectioning of the bone specimen. Finally, we could not clarify whether microdamage reduction following teriparatide treatment occurred because of increased microdamage repair or the prevention of microdamage. This is because we examined microdamage accumulation only at the end of the experiment. Time-dependent changes in microdamage parameters assessed during the time-course of teriparatide administration may help to clarify how microdamage decreases with teriparatide treatment.

Based on the results of this study, we conclude that onceweekly teriparatide treatment for 18 months improves trabecular bone mass and architecture, recovers and favorably shifts the balance of collagen cross-links, and reduces microdamage accumulation of the lumbar vertebrae of ovariectomized cynomolgus monkeys. Decreased microdamage accumulation by teriparatide treatment was associated with a decreased amount of pentosidine rather than with increased trabecular bone mass or improved trabecular architecture.
These findings suggest that once-weekly teriparatide treatment improves bone quality of the lumbar vertebrae, offering strong anti-fracture efficacy in the clinical treatment of osteoporosis.

Acknowledgements This work was funded by Asahi Kasei Pharma Corporation. The authors wish to express their appreciation to Ms. M. Higashihara and Ms. Y. Agawa for preparing undecalcified bone sections. We thank Rebecca Jackson, PhD, from Edanz Group (http:// www.edanzediting.com/ac) for editing a draft of this manuscript.

Conflict of interest Shingo Yoshitake, Tasuku Mashiba, Mitsuru Saito, Ryuji Fujihara, Ken Iwata and Tetsuji Yamamoto declare that they have no conflict of interest. Ryoko Takao-Kawabata is an employee of Asahi Kasei Pharma Corporation.

Human and Animal Rights and Informed Consent All experimental protocols were approved by the experimental animal ethics committee of Asahi Kasei Pharma Co. and were conducted in accordance with guidelines concerning the management and handling of experimental animals.

Open Access This article is distributed under the terms of the Creative Commons Attribution 4.0 International License (http://creativeco mmons.org/licenses/by/4.0/), which permits unrestricted use, distribution, and reproduction in any medium, provided you give appropriate credit to the original author(s) and the source, provide a link to the Creative Commons license, and indicate if changes were made.

\section{References}

1. Camacho PM, Petak SM, Binkley N et al (2016) American association of clinical endocrinologists and american collage of endocrinology clinical practice guideline for the diagnosis and treatment of postmenopausal osteoporosis. Endocr Pract 22:1-42. https:// doi.org/10.4158/EP161435.GL

2. Neer RM, Arnaud CD, Zanchetta JR et al (2001) Effect of parathyroid hormone (1-34) on fractures and bone mineral density in postmenopausal women with osteoporosis. N Engl J Med 344:1434-1441. https://doi.org/10.1056/NEJM200105103441904

3. Recker RR, Bare SP, Smith SY et al (2009) Cancellous and cortical bone architecture and turnover at the iliac crest of postmenopausal osteoporotic women treated with parathyroid hormone 1-84. Bone 44:113-119. https://doi.org/10.1016/j. bone.2008.09.019

4. Jiang Y, Zhao JJ, Mitlak BH et al (2003) Recombinant human parathyroid hormone (1-34) [teriparatide] improves both cortical and cancellous bone structure. J Bone Miner Res 18:1932-1941. https://doi.org/10.1359/jbmr.2003.18.11.1932

5. Dobnig H, Stepan JJ, Burr DB et al (2009) Teriparatide reduces bone microdamage accumulation in postmenopausal women previously treated with alendronate. J Bone Miner Res 24:19982006. https://doi.org/10.1359/jbmr.090527

6. Saito M, Marumo K, Kida Y et al (2011) Changes in the contents of enzymatic immature, mature, and non-enzymatic senescent cross-links of collagen after once-weekly treatment with human parathyroid hormone (1-34) for 18 months contribute to improvement of bone strength in ovariectomized monkeys. Osteoporos Int 22:2373-2383. https://doi.org/10.1007/s00198-010-1454-4

7. Chen P, Jerome CP, Burr DB et al (2007) Interrelationships between bone microarchitecture and strength in ovariectomized 
monkeys treated with teriparatide. J Bone Miner Res 22:841-848. https://doi.org/10.1359/jbmr.070310

8. Takao-Kawabata R, Isogai Y, Takakura A et al (2015) Threetimes-weekly administration of teriparatide improves vertebral and peripheral bone density, microarchitecture, and mechanical properties without accelerating bone resorption in ovariectomized rats. Calcif Tissue Int 97:156-168. https://doi.org/10.1007/s0022 3-015-9998-0

9. Jerome CP, Burr DB, Van Bibber T et al (2001) Treatment with human parathyroid hormone (1-34) for 18 months increases cancellous bone volume and improves trabecular architecture in ovariectomized cynomolgus monkeys (Macaca fascicularis). Bone 28:150-159. https://doi.org/10.1016/S8756-3282(00)00430-0

10. Nakamura $T$, Sugimoto $T$, Nakano $T$ et al (2012) Randomized teriparatide [human parathyroid hormone (PTH) 1-34] once-weekly efficacy research (TOWER) trial for examining the reduction in new vertebral fractures in subjects with primary osteoporosis and high fracture risk. J Clin Endocrinol Metab 97:3097-3106. https ://doi.org/10.1210/jc.2011-3479

11. Kim KM, Lee SY, Rhee Y (2017) Influence of dosing interval and administration on the bone metabolism,skeletal effects, and clinical efficacy of parathyroid hormone in treating osteoporosis: a narrative review. JBMR Plus 1:36-45. https://doi.org/10.1002/ jbm4.10005

12. Saito M, Fujii K, Marumo K (2006) Degree of mineralizationrelated collagen crosslinking in the femoral neck cancellous bone in cases of hip fracture and controls. Calcif Tissue Int 79:160-168. https://doi.org/10.1007/s00223-006-0035-1

13. Banse X, Sims TJ, Bailey AJ (2002) Mechanical properties of adult vertebral cancellous bone: correlation with collagen intermolecular cross-links. J Bone Miner Res 17:1621-1628. https:// doi.org/10.1359/jbmr.2002.17.9.1621

14. Tang SY, Vashishth D (2010) Non-enzymatic glycation alters microdamage formation in human cancellous bone. Bone 46:148154. https://doi.org/10.1016/j.bone.2009.09.003

15. Oxlund H, Mosekilde L, Ortoft G (1996) Reduced concentration of collagen reducible cross links in human trabecular bone with respect to age and osteoporosis. Bone 19:479-484

16. Fazzalari NL, Forwood MR, Smith K et al (1998) Assessment of cancellous bone quality in severe osteoarthrosis: bone mineral density, mechanics, and microdamage. Bone 22:381-388

17. Yamagami $Y$, Mashiba T, Iwata $K$ et al (2013) Effects of minodronic acid and alendronate on bone remodeling, microdamage accumulation, degree of mineralization and bone mechanical properties in ovariectomized cynomolgus monkeys. Bone 54:1-7. https://doi.org/10.1016/j.bone.2013.01.016

18. Mashiba T, Turner $\mathrm{CH}$, Hirano $\mathrm{T}$ et al (2001) Effects of suppressed bone turnover by bisphosphonates on microdamage accumulation and biomechanical properties in clinically relevant skeletal sites in beagles. Bone 28:524-531. https://doi.org/10.1016/ S8756-3282(01)00414-8

19. Schaffler MB (2003) Role of bone turnover in microdamage. Osteoporos Int 14(Suppl 5):S73-S77; discussion S77-80. https ://doi.org/10.1007/s00198-003-1477-1
20. Carter DR, Caler WE (1983) Cycle-dependent and time-dependent bone fracture with repeated loading. J Biomech Eng 105:166-170

21. Carter DR, Hayes WC (1976) Fatigue life of compact bone-I. Effects of stress amplitude, temperature and density. J Biomech 9:27-34

22. Burr DB (2002) Targeted and nontargeted remodeling. Bone $30: 2-4$

23. Li J, Mashiba T, Burr DB (2001) Bisphosphonate treatment suppresses not only stochastic remodeling but also the targeted repair of microdamage. Calcif Tissue Int 69:281-286. https://doi. org/10.1007/s002230010036

24. Fujita T, Inoue T, Morii H et al (1999) Effect of an intermittent weekly dose of human parathyroid hormone (1-34) on osteoporosis: a randomized double-masked prospective study using three dose levels. Osteoporos Int 9:296-306. https://doi.org/10.1007/ s001980050151

25. Saito M, Marumo K, Fujii K, Ishioka N (1997) Single-column high-performance liquid chromatographic-fluorescence detection of immature, mature, and senescent cross-links of collagen. Anal Biochem 253:26-32. https://doi.org/10.1006/abio.1997.2350

26. Dempster DW, Compston JE, Drezner MK et al (2013) Standardized nomenclature, symbols, and units for bone histomorphometry: a 2012 update of the report of the ASBMR Histomorphometry Nomenclature Committee. J Bone Miner Res 28:2-17. https://doi. org/10.1002/jbmr.1805

27. Gunness-Hey M, Hock JM (1984) Increased trabecular bone mass in rats treated with human synthetic parathyroid hormone. Metab Bone Dis Relat Res 5:177-181

28. Jerome CP, Power RA, Obasanjo IO et al (1997) The androgenic anabolic steroid nandrolone decanoate prevents osteopenia and inhibits bone turnover in ovariectomized cynomolgus monkeys. Bone 20:355-364

29. Saito M, Kida Y, Nishizawa T et al (2015) Effects of 18-month treatment with bazedoxifene on enzymatic immature and mature cross-links and non-enzymatic advanced glycation end products, mineralization, and trabecular microarchitecture of vertebra in ovariectomized monkeys. Bone 81:573-580. https://doi. org/10.1016/j.bone.2015.09.006

30. Finkelstein JS, Leder BZ, Burnett S-AM et al (2006) Effects of teriparatide, alendronate, or both on bone turnover in osteoporotic men. J Clin Endocrinol Metab 91:2882-2887. https://doi. org/10.1210/jc.2006-0190

31. McClung MR, San Martin J, Miller PD et al (2005) Opposite bone remodeling effects of teriparatide and alendronate in increasing bone mass. Arch Intern Med 165:1762-1768. https://doi. org/10.1001/archinte.165.15.1762

32. Finkelstein JS, Klibanski A, Schaefer EH et al (1994) Parathyroid hormone for the prevention of bone loss induced by estrogen deficiency. N Engl J Med 331:1618-1623. https://doi.org/10.1056/ NEJM199412153312404

33. Ming N, Cheng JT-Y, Rui Y-F et al (2012) Dose-dependent enhancement of spinal fusion in rats with teriparatide (PTH[1-34]). Spine (Phila Pa 1976) 37:1275-1282. https://doi. org/10.1097/BRS.0b013e31824ac089 